\title{
Rôle des facteurs de croissance et cytokines dans le testicule normal et pathologique
}

\author{
C. Mauduit, S. Hamamah*, B. Loras, M. Benahmed \\ INSERM U 407, Laboratoire de Recherche sur les Communications Cellulaires en Biologie \\ de la Reproduction, Bât 3 B, Centre Hospitalier Lyon-Sud, 69495 Pierre-Bénite cedex; \\ *Unité de Biologie de la Reproduction, Département de Gynéco-Obs, Reproduction Humaine \\ et Médecine Fœtale, Hôpital Bretonneau, 37044 Tours
}

\section{RESUME}

L'étude de la stérilité masculine a trouvé depuis 4 ans un regain d'intérêt avec l'avènement d'une nouvelle technique pour le traitement de l'infertilité masculine, l'injection intracytoplasmique d'un spermatozoïde dans l'ovocyte (ICSI). Néanmoins, l'étude des caractéristiques du sperme demeure le maître examen dans l'évaluation de la fertilité masculine. Il constitue le reflet de l'activité testiculaire et des événements post-gonadiques, en particulier, la maturation épididymaire. Ces processus sont pour la plupart, non seulement sous le contrôle du système neuroendocrinien, mais aussi, sous le contrôle de facteurs locaux tels que les facteurs de croissance et les cytokines. Dans cette revue, nous présentons, dans une première partie, les données montrant le rôle crucial que jouent les facteurs locaux sur la formation du testicule fotal mais aussi sur les fonctions différenciées de la gonade comme la stéroïdogenèse et la spermatogenèse. Dans une seconde partie, nous présentons les données obtenues dans le cadre de l'exploration de l'homme infertile, ainsi que les effets in vitro des facteurs locaux sur le pouvoir fécondant des spermatozoïdes. Ces dernières données sont encore fragmentaires et l'utilisation des facteurs de croissance et des cytokines dans le domaine des stérilités dites idiopathiques n'en est qu'à ses débuts. Enfin, dans le cadre d'une démarche prospective, nous indiquons quelques voies de recherche qui nous semblent intéressantes concernant l'étude des facteurs de croissance et des cytokines dans les stérilité masculines.

Mots clés : Testicule, facteurs de croissance, cytokines, oligo et azoospermie sécrétoire, infertilité masculine.

\section{INTRODUCTION}

A l'état physiologique, le spermatozoïde après sa libération de l'épithélium séminifêre subit, essentiellement sous l'influence de l'épididyme, une série de changements morphologiques et physiologiques qui conferrent aux spermatozoïdes la capacité de féconder l'ovocyte et de donner un embryon viable. Pour que la fécondation ait lieu, il faut que les spermatozoïdes reconnaissent la zone pellucide, s'y fixent, qu'ils la pénètrent et qu'il y ait fusion avec la membrane plasmique de l'ovocyte.

Ainsi deux étapes sont nécessaires pour l'élaboration d'un spermatozoïde : une étape testiculaire et une étape post-testiculaire. 
L'étape testiculaire nécessite (a) une organogenèse correcte : développement des cellules somatiques (cellules de Leydig, cellules péritubulaires myoïdes, cellules de Sertoli) et des cellules germinales (gonocytes à l'origine du futur spermatozoïde) et (b) une différenciation cellulaire pour l'acquisition des fonctions spécialisées :

- Migration cellulaire qui concerne les cellules germinales

- Prolifération cellulaire (concerne tous les types cellulaires)

- Organisation des différentes cellules somatiques et germinales entre elles pour constituer les tubes séminifères et les espaces entre ces tubes (espace interstitiel), ces deux compartiments constituant les bases anatomiques de la gonade mâle.

- Différenciation de certaines cellules somatiques qui acquièrent des fonctions très spécialisées comme la stéroïdogenèse (cellules de Leydig), mais aussi différenciation des cellules germinales qui acquièrent la mobilité et le pouvoir fécondant (spermatozoïdes).

Les bases morphologiques (tissulaire et cellulaire) de la formation du testicule ainsi que l'acquisition de ses fonctions spécifiques (stéroïdogenèse, spermatogenèse) sont connues déjà depuis longtemps. Par contre, la connaissance des bases moléculaires, en particulier la connaissance du rôle précis des molécules de signalisation impliquées dans la formation et les fonctions testiculaires, est beaucoup plus récente et connait, actuellement, un développement très important. Parmi ces molécules de signalisation importantes pour le testicule, deux familles sont identifiées : les hormones et les facteurs locaux :

- Les hormones (en particulier celles du système neurohypophysotesticulaire) constituent le contrôle de la gonade à distance. Le rôle déterminant du système endocrinien (LH-testostérone, FSH) dans la physiologie testiculaire a été démontré par un très grand nombre d'études. En pathologie, l'étude des hypofonctionnements du système endocrinien, se traduisant par un hypogonadisme, a confirmé l'importance des hormones dans le contrôle de la gonade. Bien qu'il soit indispensable pour la spermatogenèse, le système endocrinien contrôle la maturation des cellules germinales par l'intermédiaire des cellules somatiques. Ainsi, la LH régule les fonctions stéroïdogènes des cellules de Leydig et particulièrement la sécrétion de testostérone. La FSH, quant à elle, contrôle l'activité des cellules de Sertoli. La testostérone produite localement sous l'action de $\mathrm{LH}$ régule les fonctions des cellules de Sertoli et les cellules péritubulaires myoïdes. Il faut noter que les hormones LH, FSH et la testostérone ne possèdent pas de récepteurs sur les cellules germinales [17]. L'action du système endocrinien sur la maturation des gamètes nécessite donc probablement un relais local.

- Les molécules de signalisation de type facteurs de croissance et cytokines constituent le contrôle local de la gonade. Au cours de ces dernières années, un grand nombre de données ont été rapportées et indiquent le rôle potentiel de ces facteurs dans la physiologie testiculaire. Ces facteurs sont classés en différentes familles : l'Epidermal Growth Factor/Transforming Growth Factor $\alpha$ (l'EGF/TGF $\alpha)$, les Transforming Growth Factor $\beta$ (TGF $\beta$ s) et leurs peptides apparentés (activines, inhibines et l'hormone anti-mullérienne $\mathrm{AMH}$ ), les IGFs (insulin-like growth factors), les FGFs (fibroblast growth factors), les neurotrophines ( $\beta$ Nerve Growth Factor, $\beta \mathrm{NGF}$ ), les cytokines comme le Tumor Necrosis Factor $\alpha$ (TNF $\alpha$ ), l'Interleukine $1 \alpha$ (IL1 $\alpha$ ), l'Interleukine 6 (IL6), le Stem Cell Factor (SCF), le Leukemia Inhibiting Factor (LIF), les interférons (IFNs). 
Cette revue traite du rôle des facteurs de croissance et des cytokines dans le testicule, avec un double objectif :

- mettre en évidence, à travers les donnés de la littérature et nos propres travaux, le rôle potentiel de ces facteurs dans le développement et le fonctionnement testiculaire normal. Nous insisterons particulièrement sur les relations existant entre le système endocrinien et le système local constitué par les facteurs de croissance et les cytokines.

- essayer de faire le lien entre les anomalies potentielles des facteurs locaux et les situations d'infertilités, en particulier les oligo et azoospermies sécrétoires. A la différence des hormones, il est possible que ces facteurs aient aussi un rôle dans les étapes post-testiculaires dans la maturation des spermatozoïdes, notamment dans l'acquisition du pouvoir fécondant.

\section{EXPRESSION DES FACTEURS DE CROISSANCE ET DES CYTOKINES DANS LE TESTICULE : RELATION AVEC LE SYSTEME ENDOCRINIEN}

A la différence du système endocrinien, les facteurs de croissance et les cytokines agissent, en principe, sur leur lieu de production même d'où la notion d'action locale. Cette action locale permet à la cellule au sein d'un organe, d'appréhender son environnement immédiat (action autocrine) ou de communiquer avec une autre cellule (action paracrine). Ce sont ces modes de communication que l'on retrouve au sein du testicule, par exemple entre les cellules de l'espace interstitiel et les cellules du tube séminifere d'un part (communications cellules de Leydig-cellules de Sertoli) et d'autre part entre les différentes cellules du tube séminifère (communications cellules de Sertoli-cellules germinales).

Les facteurs de croissance et les cytokines ont été classés en une dizaine de famille sur les bases de leurs analogies structurales. Un autre type de classification intéressant est de les regrouper selon les systèmes de transduction qu'ils utilisent pour transmettre leur message au noyau et donc aux différents gènes de leurs cellules cibles. A titre d'exemple, on distingue les facteurs qui exercent leurs actions par l'intermédiaire de récepteurs à tyrosine kinase (ex : EGF/TGF $\alpha$, FGFs, IGFs, SCF), de récepteurs à sérine/thréonine kinase (ex : TGF $\beta$, activine, AMH). D'autres molécules de signalisation peuvent utiliser plusieurs systèmes de transduction intracellulaire ( $\mathrm{ex}$ : le TNF $\alpha$ ). Enfin, certains facteurs exercent leur action par le même récepteur et le même système de transduction (ex : EGF et $\mathrm{TGF} \alpha$ ).

Les molécules que nous décrivons sont exprimées dans différents organes. Cependant, il existe parfois des spécificités liées à l'organe où elles sont exprimés et où elles agissent. Ces molécules (ligands et récepteurs) sont présentes sous forme d'ARN messagers et de protéines dans les différents types de cellules testiculaires et à différents stades du développement gonadique (Tableau 1). L'expression de ces facteurs locaux peut être spécifique d'un type cellulaire ou d'un stade de développement testiculaire. Ainsi, certains facteurs sont produits principalement par les cellules somatiques, par exemple, le SCF et l'AMH sont produits par les cellules de Sertoli, IGF I est exprimé dans les cellules de Sertoli et les cellules de Leydig. D'autres sont exprimés surtout dans les cellules germinales comme le NGF et le TNF $\alpha$. Enfin, le TGF $\beta$ et l'EGF/TGF $\alpha$ sont exprimés de façon ubiquitaire (cellules somatiques et cellules germinales). Cette expression spécifique d'un type cellulaire ou d'un stade de développement fait intervenir des éléments de régulation transcriptionnels ou post-transcriptionnels. Par exemple, des gènes ayant des promoteurs multiples présentent une organisation propice à la régulation cloisonnée 
Tableau 1 : Expression et régulation des facteurs de croissance et des cytokines dans la gonade mâles $[4,42,67]$.

\begin{tabular}{|c|c|c|}
\hline MOLECULE & CELLULE PRODUCTRICE & REGULATION \\
\hline TGF $\beta 1$ & $\begin{array}{l}\text { Sertoli, Leydig, cellules germinales, } \\
\text { péritubulaires myoïdes }\end{array}$ & FSH $\downarrow$, T3 $\uparrow$ \\
\hline TGF $\beta 2$ & Sertoli, péritubulaires myoïdes & \\
\hline TGFß3 & Sertoli, péritubulaires myoïdes & \\
\hline $\begin{array}{l}\text { récepteurs du TGF } \beta \\
\text { (type I, II, III) }\end{array}$ & Sertoli, Leydig, cellules germinales & $\mathrm{LH} \uparrow, \mathrm{FSH} \uparrow$ \\
\hline Inhibines & Sertoli & $\begin{array}{l}\text { chaînes } \alpha: \text { FSH } \uparrow \\
\text { inhibine }: \text { EGF } \uparrow \text {, insuline } \uparrow, \\
\text { opioïds } \uparrow \text {, depletion en spermatides } \downarrow\end{array}$ \\
\hline Activines & Leydig, Sertoli, péritubulaires myoïdes & \\
\hline récepteurs des Activines & $\begin{array}{l}\text { ActRII : cellules germinales, } \\
\text { péritubulaires myoïdes, Sertoli }\end{array}$ & \\
\hline Follistatine & Sertoli, Leydig, cellules germinales & \\
\hline$\alpha 2$ macroglobuline & Sertoli & \\
\hline $\mathrm{AMH}$ & Sertoli & SF1 $\uparrow$, FSH $\downarrow$ \\
\hline récepteurs de l'AMH & Sertoli & \\
\hline TGF $\alpha$ & Sertoli, Leydig, péritubulaires myoïdes & \\
\hline EGF & cellules somatiques et germinales & \\
\hline récepteurs de l'EGF & $\begin{array}{l}\text { Sertoli, } \\
\text { Leydig, péritubulaires myoïdes, } \\
\text { cellules germinales }\end{array}$ & $\mathrm{TNF} \alpha \uparrow$ \\
\hline IGF I & Leydig, Sertoli, cellules germinales & $\begin{array}{l}\text { GH } \uparrow, \text { FSH } \uparrow, \text { LH } \uparrow, \text { Prolactine } \downarrow \text {, } \\
\text { testostérone } \uparrow, \text { rétinol } \uparrow, \text { T3 } \\
\text { EGF } \uparrow, \text { bFGF } \uparrow, \text { IL1 } \alpha \downarrow\end{array}$ \\
\hline IGF II & Sertoli, Leydig & \\
\hline IGF BP4 et 5 & cellules somatiques & \\
\hline IGF BP3 & Sertoli & $\mathrm{FSH} \downarrow, \mathrm{TNF} \alpha \uparrow, \mathrm{IL} 1 \alpha \uparrow$ \\
\hline récepteurs de l'IGF type I & Sertoli, Leydig, cellules germinales & $\mathrm{LH} \uparrow, \mathrm{TGF} \beta \downarrow$ \\
\hline récepteurs de l'IGF type II & Sertoli, cellules germinales & \\
\hline bFGF & Sertoli, Leydig, cellules germinales & FSH $\uparrow$ \\
\hline récepteurs du bFGF & Sertoli, Leydig, cellules germinales & $\mathrm{FSH} \uparrow, \mathrm{TNF} \alpha \uparrow, \mathrm{IL} 1 \alpha \uparrow, \mathrm{bFGF} \uparrow$ \\
\hline $\begin{array}{l}\text { IL1 } \\
\text { récepteurs de l'IL1 }\end{array}$ & $\begin{array}{l}\text { Sertoli, cellules germinales } \\
\text { Leydig }\end{array}$ & $\mathrm{LH} \uparrow, \mathrm{LPS} \uparrow, \mathrm{ILlb} \uparrow$ \\
\hline $\begin{array}{l}\text { IL6 } \\
\text { récepteurs de IL6 }\end{array}$ & $\begin{array}{l}\text { Sertoli, Leydig } \\
\text { Leydig, Sertoli }\end{array}$ & \\
\hline $\begin{array}{l}\text { TNF } \alpha \\
\text { récepteurs du TNF } \alpha\end{array}$ & $\begin{array}{l}\text { cellules germinales } \\
\text { Sertoli, Leydig }\end{array}$ & FSH $\uparrow$ \\
\hline $\begin{array}{l}\mathrm{SCF} \\
\text { récepteurs du SCF (c-kit) }\end{array}$ & $\begin{array}{l}\text { Sertoli } \\
\text { cellules germinales, Leydig }\end{array}$ & FSH $\uparrow$ \\
\hline NGF & cellules germinales & \\
\hline récepteurs du NGF & Sertoli, péritubulaires myoïdes & testostérone $\downarrow$ \\
\hline
\end{tabular}

$\uparrow:$ facteur dont l'expression est augmentée; $\downarrow$ : facteur dont l'expression est diminuée. 
au niveau des différents tissus (ex : le NGF utilise des promoteurs différents dans le testicule et dans le cerveau). L'épissage alternatif des préARN messagers peut faire apparaître ou non un site de protéolyse qui permet le ciblage sub-cellulaire d'une molécule (ex : production de SCF soluble ou membranaire).

Il faut noter que l'expression des facteurs locaux est modulée par les hormones (Tableau 1). Ce contrôle porte aussi bien sur les ligands et les récepteurs. Il reste cependant à déterminer le niveau de contrôle où s'exerce l'action des hormones (transcription, modification post-transcriptionnelle comme l'épissage alternatif, traduction en protéine)

\section{ACTIONS DES FACTEURS LOCAUX SUR LES FONCTIONS TESTICULAIRES}

\section{Développement gonadique fotal sous le contrôle des facteurs locaux}

Les facteurs de croissance et les cytokines ont aussi la particularité d'avoir des actions pléiotropiques, ainsi ils peuvent affecter la prolifération, la différenciation, la migration et même la mort cellulaire.

Chez l'embryon, l'individualisation de la gonade ambisexuée s'effectue lors de la colonisation de l'ébauche gonadique par les cellules germinales primordiales. Ces cellules, originaires du sac allantoïde, migrent sous l'influence de facteurs chimiotactiques. L'arrivée des cellules germinales primordiales est contemporaine de la prolifération des cellules somatiques adjacentes qui entourent les cellules germinales formant ainsi les cordons sexuels primitifs (voir revue 83 ). Le développement et la formation de la gonade vers le sexe mâle font intervenir les produits des gènes SRY (Sex Determining factor Y), DAX et SF-1 [5]. Sous l'influence de ces facteurs, des cellules somatiques des cordons sexuels primitifs se différencient en précurseurs de cellules de Sertoli. Les cellules germinales se multiplient (mais n'entrent pas en méiose) pendant la formation de la gonade fœtale puis, à la fin de la gestation, les cellules germinales entrent en quiescence jusqu'à la puberté [83].

L'étude de souris portant des mutations sur le ligand SCF (existant sous forme d'un protéine soluble et membranaire) ou son récepteur c-kit indique que la migration et la prolifération des cellules germinales seraient sous le contrôle du SCF/c-kit. Ainsi, chez les souris portant la mutation $\mathrm{Sl} / \mathrm{Sl}$ (mutation Steel : non expression du SCF, [15]) ou $\mathrm{Wv} / \mathrm{W}^{\mathrm{v}}$ (mutation dominante white-spotting : non expression du récepteur ckit, voir revue 31 ) les cellules germinales primordiales ne migrent pas dans l'ébauche gonadique. De plus, les formes solubles et membranaires du SCF n'auraient pas les mêmes propriétés. En effet, la mutation Sld (Steel Dickie : délétion du domaine transmembranaire, [6]) provoque la production de SCF sous forme soluble uniquement, les cellules germinales primordiales ont migré dans la gonade fœtale, cependant, l'animal est stérile car à la puberté, les cellules germinales ne se multiplient pas. Ces données expérimentales suggèrent que le SCF (forme soluble et surtout membranaire) aurait un rôle clé dans la migration et la prolifération des cellules de la lignée germinale. Par ailleurs, il a été montré que des cellules germinales primordiales en culture prolifêrent en présence de SCF, LIF et bFGF [19]. Ces trois molécules agiraient en synergie pour favoriser la multiplication des cellules germinales fœtales.

D'autres facteurs sont inhibiteurs de la multiplication des cellules germinales puisque le TGFb [18, 49] et l'activine A [29, 37] inhibent la multiplication des cellules germinales fœtales. Ces observations suggèrent un rôle de ces deux molécules dans l'arrêt mitotique des gonocytes vers la fin de la gestation. 
Le rôle stimulant du TGF $\beta$ sur la production des composés de la matrice extra-cellulaire indique que ce facteur pourrait intervenir dans la formation des cordons séminifères et dans la migration, la morphologie et la contractilité des cellules péritubulaires myoïdes [1].

\section{La stéroïdogenèse leydigienne sous le contrôle des facteurs locaux}

La spermatogenèse nécessite une concentration intratesticulaire très élevée en testostérone. La LH stimule la stéroïdogenèse leydigienne de façon à obtenir des concentrations en androgènes adéquates pour le maintien de la spermatogenèse. L'action de la testostérone sur le développement des cellules germinales est indirecte puisque ses récepteurs ne sont présents que sur les cellules somatiques des tubes séminifères (Sertoli, péritubulaires myoïdes). Ainsi, la testostérone pourrait agir sur les cellules germinales par l'intermédiaire de facteurs locaux produits par les cellules de Sertoli. Toute modulation de production des androgènes pourrait donc affecter la gamétogenèse. Parmi les facteurs de croissance et les cytokines qui modulent la stéroïdogenèse, on distingue des facteurs stimulants et inhibiteurs.

\section{a) Facteurs inhibiteurs :}

Plusieurs facteurs inhibent la sécrétion de testostérone induite par la LH dans les cellules de Leydig : le TGF $\beta[34,46]$, l'activine A $[25,39]$, l'IL $1 \alpha[7,41]$ et le TNF $\alpha[8,40$, $44,84]$. Cependant, ces facteurs n'agissent pas sur les mêmes étapes biochimiques de la stéroïdogenèse. Ainsi, les cytokines (TNF $\alpha$, IL $1 \alpha$ ) inhibent le transport vers les mitochondries et la mise à disposition du cholestérol pour le cytochrome P450 de la desmolase mitochondriale $[40,41]$. Le TGF $\beta$ et l'activine A inhibent le cytochrome P450 de la desmolase [3, 35, 39, 76] (Figure 1).

Ces différents facteurs agiraient probablement à différents moments de la vie de la gonade. En effet, le TGFb et l'activine A sont présents avant la puberté et leur expression décroît pendant la puberté $[4$, 42]. Le TNF $\alpha$ n'interviendrait qu'à l'âge adulte puisque cette molécule est sécrétée par les cellules germinales et les macrophages testiculaires [4, 42].

\section{b) Facteurs stimulants}

Les facteurs stimulants de la stéroïdogenèse induite par la LH sont l'inhibine [25], l'EGF [48, 75, 78, 82], IGF I [2, 33] et le basic FGF [74]. Il a été montré que l'EGF testiculaire et plasmatique contrôlent la production de testostérone puisque une sialoadénalectomie chez la souris provoque une diminution des taux plasmatiques de testostérone et de FSH et un hypogonadisme $[57,68,80]$.

En ce qui concerne les mécanismes d'action de ces molécules (Figure 2), l'EGF augmente la sécrétion de testostérone en activant le transport du cholestérol et l'activité $3 \beta$ hydrostéroïde déshydrogénase $[75,76]$. L'IGF I agit sur différentes étapes : il augmente le nombre de récepteurs de LH, l'activité de l'adénylate cyclase couplée au récepteur de $\mathrm{LH}$, il augmente l'expression du cytochrome $\mathbf{P} 450$ de l'enzyme desmolase et l'activité $3 \mathrm{~b}$ hydrostéroïde déshydrogénase $[2,33]$. Etant donné que la LH favorise l'expression d'IGF I dans la gonade, ce facteur semble être un relais privilégié de l'action de la gonadotrophine sur les cellules de Leydig (amplificateur local de l'action de LH). Quant au bFGF, les études publiées sont contradictoires. Dans certains modèles, il stimule la fonction stéroïdogène [74] et dans d'autres, il l'inhibe [14, 47]. Cependant, il y a un relatif consensus sur l'action stimulante du bFGF sur la prolifération des cellules de Leydig dans la période ante et postnatale [20].

En somme, en plus du rôle de relais de l'action hormonale joué par les facteurs locaux, il semblerait que ces molécules interferrent, au niveau du testicule, avec le système 


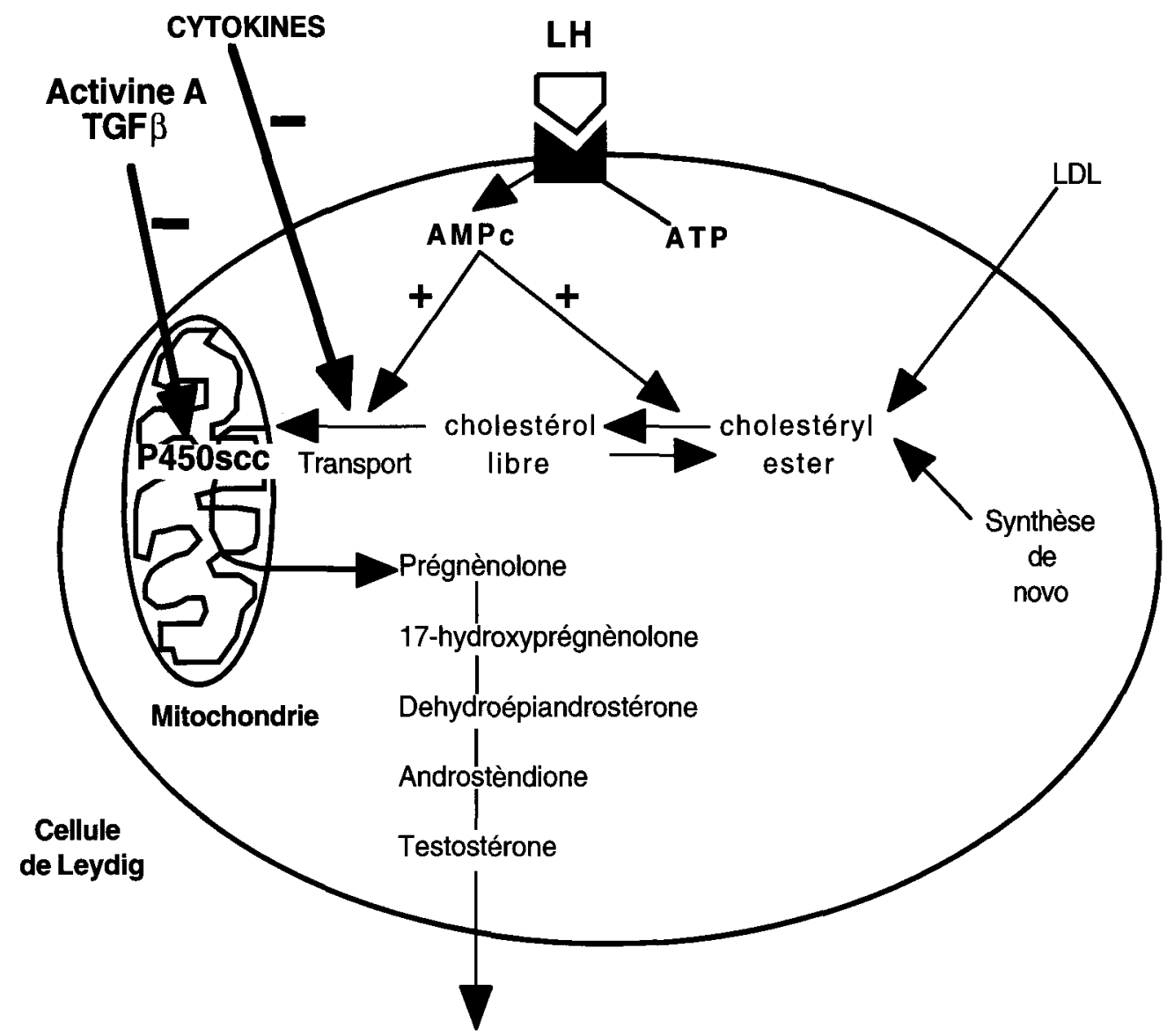

Figure 1 : Sites d'action des facteurs de croissance et des cytokines inhibiteurs sur la stéroüdogenèse leydigienne.

endocrinien en modulant l'action de la $\mathrm{LH}$ et de la FSH. Ces observations suggèrent l'existence de boucles courtes de régulation intra-testiculaires entre les hormones et les facteurs de croissance intervenant dans le contrôle de certaines fonctions testiculaires.

\section{Spermatogenèse sous le contrôle des facteurs locaux}

a) Action directe sur les cellules germinales

Les étapes fondamentales de la spermatogenèse comme la mitose, la méiose, la différenciation et la mort des cellules germinales, pourraient être sour le contrôle direct de certains facteurs de croissance et cytokines produits par les cellules de Sertoli.
En effet, les cellules germinales expriment des récepteurs spécifiques pour des molécules produites par les cellules de Sertoli comme EGF/TGF $\alpha$, les TGF $\beta$ et ses peptides apparentés, le SCF, l'IGF I et le bFGF $[4,42,67]$ (Tableau 1). Ces facteurs pourraient contrôler la survie (SCF, [38]), la multiplication des spermatogonies (EGF/TGF $\alpha$, bFGF, IGF I ; [23, 30, 61, 64, 73]), l'arrêt de la multiplication de ces cellules (TGFb, [18] ; inhibine, [16, 81] ; IL6, [22]), la méiose (EGF, TGF $\beta$, bNGF ; [62]) et la spermiogenèse (EGF).

De plus, ces facteurs sertoliens qui contrôlent la spermatogenèse, peuvent être régulés par les hormones. Ces observations illustrent, de nouveau, le rôle de relais local des molécules 


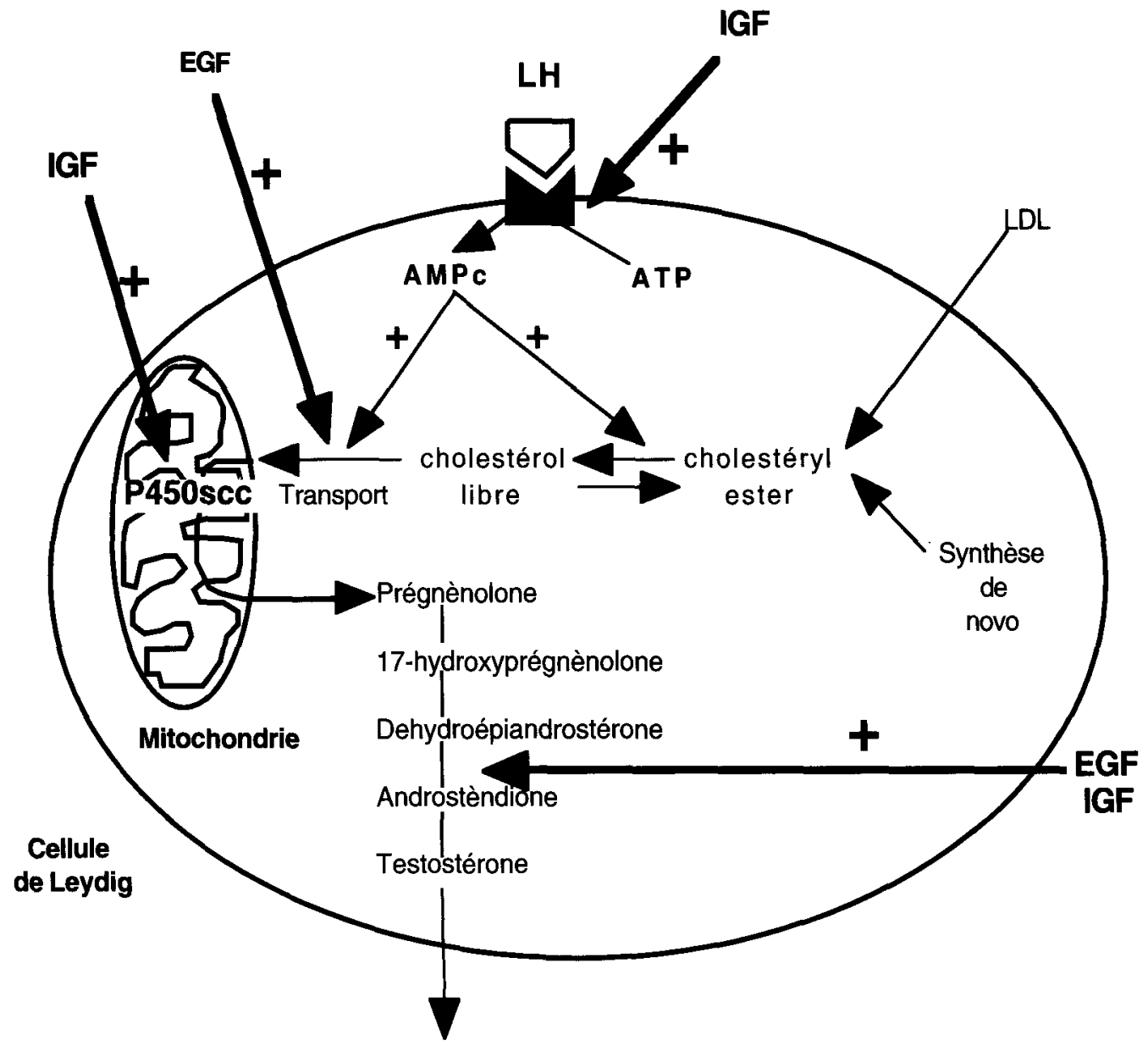

Figure 2 : Sites d'action des facteurs de croissance et des cytokines stimulants sur la stéroüdogenèse leydigienne.

de signalisation vis-à-vis des hormones (FSH, testostérone) (Figure 3). Cependant, l'expression de ces facteurs sertoliens peut être aussi sous le contrôle de facteurs locaux comme le TNF $\alpha$, l'IL1 $\alpha$ ou le NGF, provenant des cellules germinales. L'ensemble de ces observations indiquent clairement que les facteurs de croissance et les cytokines joueraient un rôle de médiateurs dans les interactions cellules de Sertoli-cellules germinales.

\section{b) Action indirecte via les cellules de Sertoli}

- Par l'intermédiaire d'éléments nutritifs comme le lactate

Les facteurs locaux favorisent le développement des cellules de la lignée germinale en augmentant la production des métabolites énergétiques comme le lactate. En effet, les cellules de la lignée germinale ne métabolisent pas le glucose mais utilisent préférentiellement le lactate provenant des cellules de Sertoli [54]. La FSH, l'insuline mais aussi des facteurs locaux comme IGF I, EGF augmentent la production de lactate par les cellules de Sertoli $[36,45,54,59]$. Ces molécules stimulent la production de lactate en favorisant, à court terme, la captation de glucose (principalement en augmentant les transporteurs de glucose). D'autres facteurs comme le TGF $\beta$, le TNF $\alpha$ et l'IL1 $\alpha$ augmentent la production de lactate à long terme [55]. Le TNF $\alpha$ et l'IL1 $\alpha$ élèvent les taux de lactate par au moins deux types de mécanismes, en 


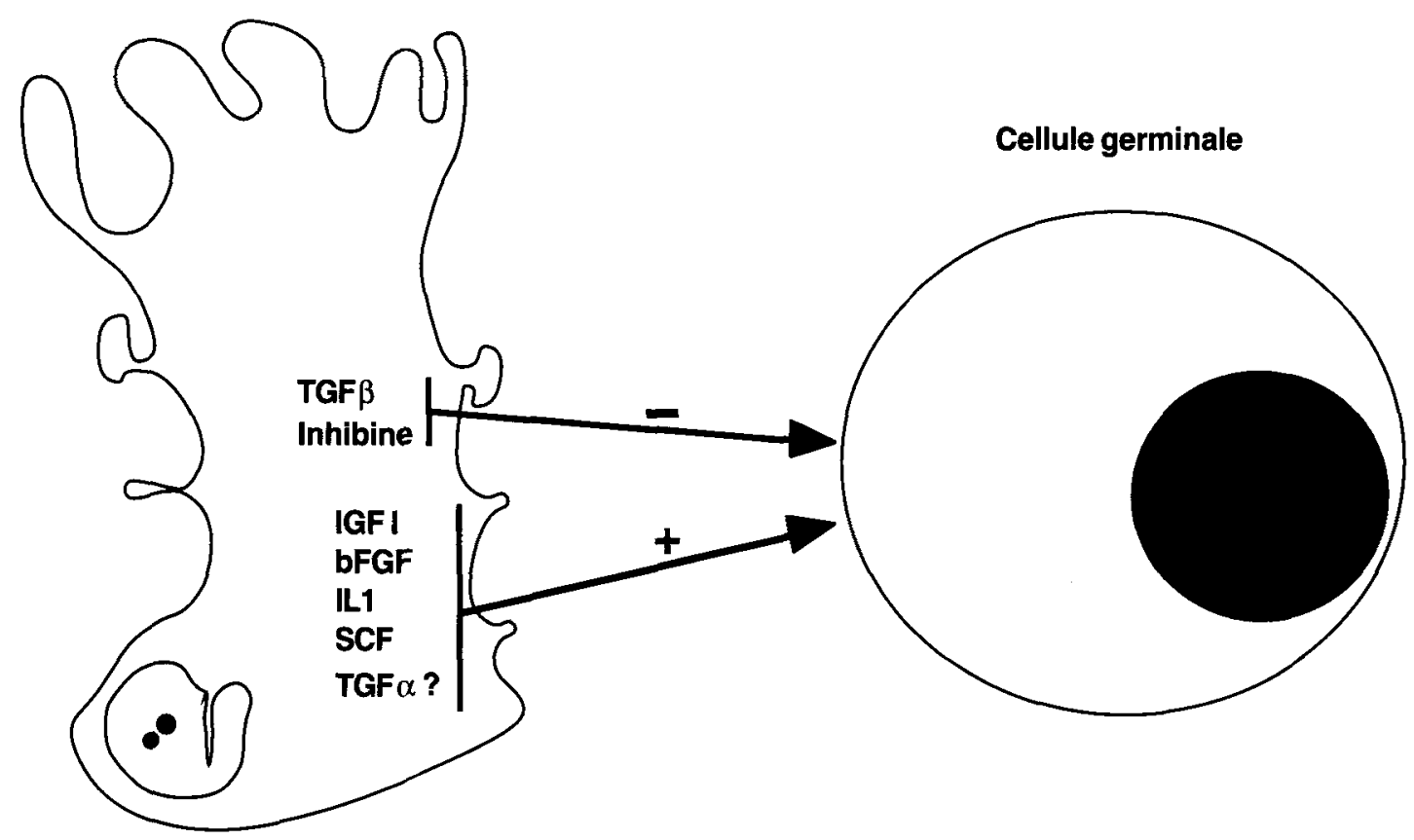

Figure 3 : Facteurs de croissance et cytokines produits par les cellules de Sertoli sous le contrôle des hormones (FSH) et potentiellement impliqués dans la maturation des cellules germinales.

augmentant l'expression et l'activité de l'enzyme lacticodéshydrogénase plus particulièrement l'isoforme 5 (qui transforme le lactate en pyruvate) et la captation de glucose [55].

- Par l'intermédiaire du nombre de cellules de Sertoli

Le nombre de cellules de Sertoli présentes dans le testicule, dès la vie fœetale, conditionne le nombre de cellules germinales présentes à l'âge adulte. Autrement dit, tous les facteurs capables de stimuler ou d'inhiber la prolifération des cellules de Sertoli affecteront la production des gamètes [71]. Plusieurs facteurs ont été identifiés comme ayant un rôle positif dans la prolifération des cellules de Sertoli fotales : le bFGF, l'IGF I et l'EGF.

\section{APPLICATION CLINIQUE DE L'EVA- LUATION DES FACTEURS LOCAUX TESTICULAIRES}

Les facteurs de croissance et les cytokines peuvent jouer un rôle important (a) dans la formation du spermatozoïde, (b) dans l'acquisition de son pouvoir fécondant, (c) dans sa protection vis-à-vis du système immunitaire mâle (qui ne le reconnaît pas comme du "soi") et femelle et enfin, (d) au moment de l'interaction avec la zone pellucide de l'ovocyte.

Compte tenu du rôle potentiellement important des facteurs de croissance et des cytokines dans la physiologie testiculaire, deux questions se posent lorsque nous abordons la pathologie testiculaire et en particulier dans les oligo et les azoospermies sécrétoires dont la cause n'est pas clairement identifiée [79] :

- Dans une démarche diagnostic, existe-t-il des modifications de la production de ces molécules de signalisation dans les oligo et les azoospermies sécrétoires ? En d'autres termes, le niveau d'expression dans le tissu testiculaire, les taux plasmatiques et les concentrations dans le 
liquide séminal de ces molécules peuventils être utilisés comme marqueurs de ces pathologies et plus spécifiquement, comme marqueurs de la perturbation d'un type de cellules testiculaires?

- Dans une démarche thérapeutique, ces molécules peuvent-elles être utilisées pour corriger certains troubles de la spermatogenèse et/ou le pouvoir fécondant du spermatozoïde?

\section{Exploration des facteurs de croissance et des cytokines chez l'homme infertile}

Les molécules de signalisation peuvent être recherchées au moins à trois niveaux différents : le sang, le plasma séminal et le tissu testiculaire.

\section{a) Le sang}

En principe, les facteurs de croissance et les cytokines à la différence des hormones ne sont pas présents dans le sang puisque leur action est de type local (auto/paracrine) c'està-dire qu'ils agissent à l'endroit même où ils sont sécrétés. Cependant, certains facteurs comme l'IGF I, les peptides apparentés du TGF $\beta$ (ex : les inhibines) ou même l'EGF (en particulier dans le modèle murin) se comporteraient aussi comme des hormones puisqu'ils sont retrouvés dans le sang, dans des conditions physiologiques $[43,68]$. Cependant, l'origine multiple (production dans différents organes) de ces facteurs fait que pour beaucoup, l'évaluation de leur taux sanguin ne reflète en aucun cas l'activité (d'un type cellulaire) du testicule. Pour certains peptides apparentés du TGF $\beta$, en particulier l'inhibine $\mathrm{B}$ (dimère $\alpha \beta \mathrm{B}$ ), différents travaux [43] semblent indiquer que, son taux plasmatique pourrait refléter l'activité des cellules de Sertoli. Si ces observations se confirmaient, elles présenteraient un intérêt majeur dans la mesure où elles permettraient de différencier les oligo et les azoospermies sécrétoires des oligo et des azoospermies excrétoires lorsque les taux plasmatiques de FSH ne peuvent le permettre [58].

\section{b) Le plasma séminal}

Le plasma séminal constitue le milieu biologique où l'on retrouve les sécrétions testiculaires, épididymaires mais surtout celles des vésicules séminales et de la prostate. En conséquence, la mise en évidence dans le plasma séminal d'un facteur de croissance ou d'une cytokine doit tenir compte de ces origines multiples. De nombreuses molécules de signalisation ont été identifiées dans le plasma séminal (Tableau 2).

Peut-on corréler les taux de ces facteurs avec les différentes étiologies des oligo et azoospermies ? Des précautions doivent être prises quant à l'origine testiculaire ou extra-testiculaire et quant aux types de dosages utilisés (RIA, ELISA, bioactivité). Aussi, les dosages utilisés doivent tenir compte de la nature protéique des facteurs de croissance et des cytokines. En effet, un anticorps donné (dans un dosage immunologique donné) reconnaît une (des) séquence(s) polypeptidique(s), ce qui peut expliquer des variations de résultats importantes entre les différents kits (c'est-à-dire les différents anticorps) du commerce. Par ailleurs, un anticorps reconnaît dans le peptide une (des) séquence(s) d'acides aminés qui ne reflètent pas forcément l'activité biologique de ce peptide. Dans ces cas, seuls des dosages de bioactivité (utilisant par exemple des lignées cellulaires avec un marqueur spécifique du peptide étudié) ou un dosage par radio-récepteur peuvent valider le dosage immunologique. Par ailleurs, cette approche devrait aussi permettre de sélectionner parmi les kits du commerce (qui sont de plus en plus nombreux), ceux qui donnent des valeurs les plus proches de celles du dosage biologique.

Quelques travaux rapportent des variations du taux des facteurs de croissance et des cytokines dans le plasma séminal de patients stériles $[11,28,52,56]$. Pour la grande majorité de ces facteurs, et en particulier pour les cytokines, il semble que leurs 
Tableau 2 : Valeurs des facteurs de croissance et des ctyokines dans le plasma séminal.

\begin{tabular}{|c|c|c|c|c|}
\hline \multirow[t]{2}{*}{ FAMILLE } & \multirow{2}{*}{$\begin{array}{l}\text { MOLECULE } \\
\text { DOSEE }\end{array}$} & \multicolumn{2}{|c|}{ PLASMA SEMINAL } & \multirow[t]{2}{*}{ Réf. } \\
\hline & & Normal & Pathologique & \\
\hline \multirow[t]{6}{*}{ IGF } & IGF 1 & $20 \mathrm{ng} / \mathrm{ml}$ & $\downarrow$ après vasectomie & 60,66 \\
\hline & IGF 2 & $2000 \mathrm{ng} / \mathrm{ml}$ & & 66 \\
\hline & IGF BP1 & $0,95 \pm 0,4 \mathrm{ng} / \mathrm{ml}$ & $\begin{array}{l}\text { non modifiée dans l'azoo- } \\
\text { spermie et après vasectomie }\end{array}$ & 60 \\
\hline & IGF BP2 & présente & $\begin{array}{l}\text { non modifiée dans l'azoo- } \\
\text { spermie et après vasectomie }\end{array}$ & 32,60 \\
\hline & IGF BP3 & $845 \pm 59 \mathrm{ng} / \mathrm{ml}$ & $\downarrow$ après vasectomie & 60 \\
\hline & IGF BP4 & présente & $\begin{array}{l}\text { non modifiée dans l'azoo- } \\
\text { spermie et après vasectomie }\end{array}$ & 32,60 \\
\hline \multirow[t]{3}{*}{ TGF $\beta$} & TGF $\beta 1$ latent & $90 \mathrm{ng} / \mathrm{ml}$ & $\begin{array}{l}\text { TGF } \beta \uparrow \text { dans stérilité } \\
\text { sécrétoire et surtout quand } \\
\text { il y a eu infection }\end{array}$ & $*$ \\
\hline & TGF $\beta 1$ libre & $2 \mathrm{ng} / \mathrm{ml}$ & & $*$ \\
\hline & inhibine $\alpha$ et $\beta$ & présentes & & 43 \\
\hline $\mathrm{EGF} / \mathrm{TGF} \alpha$ & EGF & $20-80 \mathrm{ng} / \mathrm{ml}$ & $\begin{array}{l}\text { pas de corrélation entre } \\
\text { taux EGF et paramètres } \\
\text { concernant les spermatozoïdes }\end{array}$ & $\begin{array}{l}10,13 \\
50,63 \\
86, *\end{array}$ \\
\hline \multirow[t]{4}{*}{ cytokines } & $\operatorname{IL} 1 \beta$ & $\begin{array}{l}\text { non détéctée ou } \\
40 \mathrm{ng} / \mathrm{ml}\end{array}$ & $\begin{array}{l}\text { dans oligo et azoospermie, } \\
\text { non detectée ou taux normaux. } \\
\uparrow \text { après infection } \\
\text { Pas de corrélation avec morpho- } \\
\text { logie ou mobilté du spermatozoïde }\end{array}$ & $\begin{array}{l}9,21,27, \\
51\end{array}$ \\
\hline & $\begin{array}{l}\text { antagoniste } \\
\text { récepteur IL1 }\end{array}$ & $120 \pm 10 \mathrm{pg} / \mathrm{ml}$ & $\begin{array}{l}\text { taux } \uparrow \text { dans l'azoospermie } \\
\text { surtout si associée à une infection }\end{array}$ & 26,27 \\
\hline & $\mathrm{IL} 2$ & $15 \mathrm{pg} / \mathrm{ml}$ & $\uparrow$ lors d'infection & 65,72 \\
\hline & $\begin{array}{l}\text { récepteur } \\
\text { soluble IL2 }\end{array}$ & $410 \mathrm{UI} / \mathrm{ml}$ & $\begin{array}{l}\downarrow \text { dans les oligo et azoospermies } \\
\text { associées à une infection }\end{array}$ & 26 \\
\hline \multirow[t]{8}{*}{ Cytokines } & IL6 & $5-80 \mathrm{pg} / \mathrm{ml}$ & $\begin{array}{l}\uparrow \text { après immuno-infection pas } \\
\text { de corrélation avec le nombre, } \\
\text { la mobilité des spermatozoïdes }\end{array}$ & $\begin{array}{l}9,21 \\
51,72\end{array}$ \\
\hline & $\begin{array}{l}\text { récepteur soluble } \\
\text { IL6 }\end{array}$ & $16 \mathrm{ng} / \mathrm{ml}$ & non modifié & 9 \\
\hline & IL8 & $1 \mathrm{ng} / \mathrm{ml}$ & $\uparrow$ lors de leucospermies & 66,72 \\
\hline & interféron $\gamma$ & $0,25 \mathrm{UI} / \mathrm{ml}$ & non modifié & 51 \\
\hline & TNF $\alpha$ & $\begin{array}{l}\text { non détécté à } \\
8 \mathrm{pg} / \mathrm{ml}\end{array}$ & $\begin{array}{l}\uparrow \text { dans azoospermie après } \\
\text { infection bactérienne ou à } \\
\text { mycoplasme }\end{array}$ & $\begin{array}{l}21,27 \\
51,\end{array}$ \\
\hline & $\begin{array}{l}\text { récepteur soluble } \\
\text { TNF } \alpha \text { p55 }\end{array}$ & $4-60 \mathrm{ng} / \mathrm{ml}$ & $\begin{array}{l}\text { non modifié dans l'azoospermie } \\
\text { ou après vasectomie, } \downarrow \text { lors } \\
\text { d'infection du tractus génital }\end{array}$ & 27,77 \\
\hline & $\begin{array}{l}\text { récepteur soluble } \\
\text { TNF } \alpha \text { p75 }\end{array}$ & non détecté & & 27,77 \\
\hline & SCF & dectecté & & 70 \\
\hline
\end{tabular}

$\uparrow$ augmentation du taux $d u$ facteur ; $\downarrow$ diminution du taux du facteur ; * nos travaux. 
taux varient en fonction de l'état infectieux du tractus génital (Tableau 2). En ce qui concerne les facteurs de croissance, les travaux sont encore rares et seules des précautions minutieuses devraient permettre d'utiliser ces molécules comme marqueur de l'état fonctionnel de la spermatogenèse.

\section{c) Le tissu testiculaire}

La recherche de molécules de signalisation au niveau du tissu testiculaire fait appel à différentes techniques permettant d'identifier le signal au niveau du mRNA (hybridation in situ, RT-PCR, northern blotting) et/ou de la protéine (western blotting, immunohistochimie). Dans ce contexte, différentes molécules de signalisation comme IGF I, EGF , SCF, c-kit [4, 42,67] ont été identifiées, en général par des techniques d'immunohistochimie. Compte tenu des faibles quantités de tissus testiculaires obtenus, la RT-PCR pourrait s'avérer une technique intéressante pour :

- identifier les messagers recherchés et leurs récepteurs

- identifier des anomalies de type délétion, mutations, modification de l'épissage alternatif. La RT-PCR pourrait être intéressante pour une approche visant à détecter plutôt des anomalies qualitatives que quantitatives. En effet, une augmentation ou une diminution du niveau d'expression d'une molécule de signalisation ne signifie pas pour autant une atteinte quelconque puisqu'à l'état physiologique, le niveau d'expression varie de façon considérable dans le cadre d'interactions cellulaires et en particulier entre les cellules de Sertoli et les cellules germinales au cours des différentes phases de la spermatogenèse.

\section{Action in vitro des facteurs de crois- sance et des cytokines sur le sperme provenant de patients infertiles}

A l'état physiologique, à la sortie du testicule, les spermatozoïdes sont incapables de féconder l'ovule. Ils acquièrent leur pour- voir fécondant lorsqu'ils traversent le canal épididymaire. Pour que la fécondation ait lieu, il faut que les spermatozoïdes reconnaissent la zone pellucide, s'y fixent, qu'ils la pénètrent et qu'il $\mathrm{y}$ ait fusion avec la membrane plasmique de l'ovocyte.

Différents travaux indiquent qu'au delà du rôle des cytokines sur la formation des spermatozoïdes, ces molécules peuvent affecter l'état fonctionnel des gamètes. TGFb, IL1, IL2, GM-CSF se sont révélés sans effets particuliers sur la fonction des spermatozoïdes [24, 49]. Par contre, l'interféron a et $t$ ainsi que le TNF $\alpha$ ont un effet délétère sur la mobilité des spermatozoïdes ainsi que sur la pénétration dans la zone pellucide de l'ovocyte $[12,53]$. Cependant, le traitement des hommes stériles par l'IFN a permis d'améliorer le nombre et la mobilité des spermatozoïdes [85]. Bien que ce travail paraisse prometteur, d'autres travaux demeurent nécessaires pour comprendre, en particulier, l'apparente contradiction entre les observations obtenues in vivo et in vitro.

Les facteurs de croissance ayant des récepteurs à tyrosine kinase mais probablement aussi à sérine thréonine kinase pourraient s'avérer intéressants dans le pourvoir fécondant du spermatozoïde et au moment de l'interaction inter-gamétique. Parmi les facteurs de croissance à récepteurs tyrosine kinase d'intérêt, l'EGF/TGF $\alpha$, les récepteurs de l'EGF sont présents sur les spermatozoïdes où ils sont fonctionnels. Naz et al. [50] ont observé une absence d'effet in vitro de l'EGF sur le pouvoir fécondant du spermatozoïde (en présence de faibles concentrations d'EGF) alors qu'ils observent en présence de fortes concentrations d'EGF un effet inhibiteur sur la mobilité du spermatozoïde, sur le taux de pénétration des ovocytes (en Hamster test), sur la réaction acrosomique spontanée ou induite par le calcium ionophore. Ces résultats devront être confirmés. Les mêmes auteurs ont utilisé de l'EGF recombinant dont la bioactivité au niveau du plasma séminal doit être validé. 
Tableau 3 : Effets du retrait ou de la surexpression des facteurs locaux sur la spermatogenèse.

MOLECULE

TGF $\beta$ (facteur inhibiteur de la spermatogenèse)

IGF I (facteur stimulant la spermatogenèse)

SCF ou son récepteur c-kit (système stimulant la spermatogenèse)

interféron $\gamma$

TYPE DE MUTATION CONSEQUENCES

surexpression

retrait (knock-out)

délétion

surexpression stérilité [69]

stérilité [42]

stérilité [42]

stérilité [42]
Enfin, l'IL6 apparaît comme une des molécules intéressantes dans le cadre du traitement de la stérilité masculine, puisque cette cytokine a été détectée dans le plasma séminal d'hommes infertiles à des taux élevés (infections?). Son action sur le pouvoir fécondant des spermatozoïdes in vitro présente quelques intérêts. En effet, l'IL6 semble améliorer le pourcentage de formes mobiles et augmenter la réaction acrosomique spontanée ou stimulée par le calcium ionophore.

\section{PROSPECTIVES}

Différentes étapes cruciales nécessaires à l'obtention de spermatozoïdes fonctionnels semblent être en relation avec les activités pléiotropiques des facteurs de croissance et des cytokines comme (a) la formation et le développement de la gonade foetale, (b) la mise en place de la spermatogenèse à la puberté, (c) l'acquisition du pouvoir fécondant dès la sortie du testicule, (d) la protection des spermatozoïdes vis-à-vis des systèmes immunitaires mâle et femelle et enfin (e) l'interaction avec le gamète femelle. A la différence du système endocrinien, qui agit à distance, les facteurs de croissance et les cytokines constituent un système de contrôle local c'est-à-dire que ces facteurs exercent leurs activité biologique là où ils sont produits. Il existe des interactions étroites entre le système endocrinien et le système local. D'une part, en contrôlant l'expression des fac- teurs de croissance et des cytokines (et aussi de leurs récepteurs) dans le testicule, le système endocrinien pourrait utiliser le système local comme un relais, en particulier pour réguler la spermatognèse (mitose, méiose, différenciation et apoptose). D'autre part, les facteurs locaux peuvent à leur tour amplifier ou inhiber l'action des hormones et ce probablement afin d'adapter finement l'action hormonale aux besoins de la spermatogenèse. Il est tout à fait possible que les interactions hormones-facteurs locaux soient beaucoup plus importantes lors de la formation des gamètes (étape testiculaire) plutôt que lors de l'acquisition du pouvoir fécondant (étape post-testiculaire).

Ainsi des anomalies potentielles affectant l'expression et/ou l'action des facteurs locaux à l'une de ces étapes, pourraient entraîner une situation d'infertilité. Les anomalies pourraient, en particulier, rendre inefficace le système endocrinien dont l'action est décisive pour la spermatogenèse. D'ailleurs des données expérimentales de transgenèse (knock-out ou surexpression) montrent qu'une expression inadéquate de facteurs de croissance ou des cytokines peut aboutir à la stérilité (Tableau 3). Sachant qu'un très grand nombre d'infertilités, en particulier les oligo et les azoospermies sécrétoires, restent encore inexpliquées, il apparait donc important d'essayer d'utiliser les connaissances acquises sur ces facteurs pour tenter d'expliquer ces pathologies testiculaires. 
Ces molécules sont recherchées dans le tissu testiculaire, le plasma sanguin et le plasma séminal. Le tissu testiculaire offre peut-être les possibilités les plus intéressantes pour essayer d'identifier différents types d'anomalies entraînant des pertes de fonctions du ligand, du récepteur, du système de transduction ou des facteurs de transcription utilisés par les facteurs locaux pour contrôler l'expression de leurs gènes cibles. Le plasma sanguin pourrait s'avérer intéressant pour détecter des molécules de signalisation provenant de cellules de Sertoli. L'exemple de l'inhibine B pourrait être suivi par la mise en évidence d'autres marqueurs intéressants. Enfin, le plasma séminal, qui est le résultat des sécrétions gonadiques et extragonadiques, pourrait permettre d'identifier des molécules marqueurs des différents types d'oligo et d'azoospermies sécrétoires et excrétoires. Dans ces cas, la coopération entre le médecin, le biologiste et le chercheur est décisive pour distinguer et classer les différents types d'oligo et d'azoospermies. Les facteurs de croissance et les cytokines étant avant tout des peptides, leur manipulation et identification dans un milieu complexe comme le plasma séminal, requiert néanmoins énormément de précautions si l'on ne veut pas s'exposer à des risques de mauvaises exploitations/interprétations des données obtenues.

\section{REFERENCES}

1. Ailenberg M., Tung P.S., Fritz I.B. : Transforming growth factor-beta elicits shape changes and increases contractility of testicular peritubular cells. Biol. Reprod., 1990, 42 : 499-509.

2. Benahmed M., Morera A.M., Chauvin M.A., de PERETTI E. : Somatomedin C/insulin-like growth factor 1 as a possible intratesticular regulator of Leydig cell activity. Mol. Cell Endocrinol., 1987, $50: 69-77$.

3. Benahmed M., Sordoillet C., Chauvin M.A., De Peretti E., Morera A.M. : On the mechanisms involved in the inhibitory and stimulating actions of Transforming Growth Factor b on porcine testicular steroidogenesis : an in vitro study. Mol. Cell Endocrinol., 1989, $67:$ 155-164.
4. Benahmed M. : Growth factors and cytokines in the testis. In : F. Comhaire. Male infertility: Clinical investigation, cause, evaluation and treatment. London, Chapman Hale, 1996 : pp 55-96

5. Berta P., Hawkins J.R., Sinclair A.H., ET AL. : Genetic evidence equating SRY and the testis determining factor. Nature, $1990,348: 448-450$.

6. BRanNan C.I., Lyman S.D., Williams D.E., ET AL. : Steel-Dickie mutation encodes a c-kit ligand lacking transmembrane and cytoplasmic domains. Proc. Natl. Acad. Sci. U. S. A., 1991, 88 : 46714674.

7. Calkins J.H., Sigel M.M., Nankin H.R., Lin T. : Interleukin-1 inhibits Leydig cell steroidogenesis in primary culture. Endocrinology, 1988, 123 : 1605-1610.

8. Calkins J.H., Guo H., Sigel M.M., Lin T. : Tumor necrosis factor-a enhances inhibitory effects of interleukin-1b on Leydig cell steroidogenesis. Biochem Biophys Res Commun, 1990, 166 : 1313-1318.

9. Comhaire F., Bosmans E., Ombelet W., Punjabi U., ScHOONJANS F. : Cytokines in semen of normal men and of patients with andrological diseases. Am. J. Reprod. Immunol., 1994, 31 : 99-103.

10. D'CRUZ O.J., HAAS JR G.G. : Immunoreactive human epidermal growth factor in human seminal plasma. J. Clin. Endocrinol. Metab., 1989, 68 : 1136-1140.

11. Depuydt C.E., Bosmans E., Zalata A., Schoonjans F., ComhalRe F.H. : The relation between reactive oxygen species and cytokines in andrological patients with or with out male accessory gland infection. J. Androl., 1996, 17 : 699-707.

12. Eisermann J., Register K.B., StRickler R.C., ColLIN J.L. : The effect of tumor necrosis factor on human sperm motility in vitro. J. Androl., 1989, $10: 270-274$.

13. Elson S.D., Browne C.A., ThorbuRn G.D. : Identification of epidermal growth factor-like activity in human male reproductive tissues and fluids. J. Clin. Endocrinol, Metab., 1984, 58 : 589-594.

14. Fauser B.C., Baird A., Hsueh A.J. : Fibroblast growth factor inhibits luteinizing hormone-stimulated androgen production by cultured rat testicular cells. Endocrinology, 1988, 123 : 2935-2941.

15. Flanagan J.G., Chan D., Leder P. : Transmembrane form of the c-kit ligand growth factor is determined by alternative splicing and is missing in the Sld mutation. Cell, 1991, 64 : 1025-1035.

16. Franchimont P., Croze F., Demollin A., Bologne R., Hustin J. : Effect of inhibin in rat testicular deoxyribonucleic acid (DNA) synthesis in vitro. Acta Endocrinol. Copenh., 1981, 98 : 312-320. 
17. FRITZ I.B. : Sites of action of androgens and follicle stimulating hormones on cells of the seminiferous tubule. In : G. Litwack. Biochemical Actions of Hormones,. New York, Academic Press, 1978 : 249281

18. GodIN I., WYLIE C.C. : TGF $\beta 1$ inhibits proliferation and has a chemotropic effect on mouse primordial germ cells in culture. Development, 1991, 113 : 1451-1457.

19. Godin I., Deen R., Cooke J., Zsebo K., Dexter M., WYLIE C.C. : Effects of the steel gene product on mouse primordial germ cells in culture. Nature, 1991, 352 : 807-809.

20. Gonzales A.-M., Buscaglia M., Ong M., BaiRd A. : Distribution of basic fibroblast growth factor in 18. day-old rat fetus: localization in basement membranes of diverses tissues. J. Cell Biol., 1990, 110 ; 753-765.

21. Gruschwitz M.S., Brezinschek R., BrezinscheK H.-P. : Cytokine levels in the seminal plasma of infertile males. J. Androl., 1996, 17 : 158-163.

22. Hakovirta H., Syed V., Jegou B., Parvinen M. : Function of interleukin- 6 as a inhibitor of meiotic DNA synthesis in the rat seminiferous epithelium. Mol. Cell Endocrinol., 1995, 108 : 193-198.

23. Haneji T., Koide S.S., Tajima Y., Nishimune Y. : Differential effects of epidermal growth factor on the differentiation of type A spermatogonia in adult mouse cryptorchid testes in vitro. J. Androl., $1991,12: 383-388$.

24. Haney A.F., Hughes S.F., Weinderg J.B. : The lack of effect of tumor necrosis factor-alpha, interleukin1-alpha, and interferon-gamma on human sperm motility in vitro. J. Androl., 1992, 13 : 249-253.

25. Hsueh A.J.W., Dahl K.D., Vaughan J., et al. : Heterodimers and homodimers of inhibin subunits have different paracrine action in the modulation of luteinizing hormone-stimulated androgen biosynthesis. Proc. Natl. Acad. Sci. U. S. A., 1987, 84 : $5082-5086$

26. Huleihel M., Lunenfeld E., Ayelet L., PostashNIK G., GLEZERMAN M. : Cytokine expression in seminal plasma of fertile and infertile males. 11th Annual Meeting of the European Society of Human Reproduction and Embryology. Hamburg, 1995.

27. Huleihel M., Lunenfeld E., Ayelet L., Potashnik G., Glezerman M. : Distinct expression levels of cytokines and soluble cytokine receptors in seminal plasma of fertile and infertile men. Fertil. Steril., 1996, 66 : 135-139.

28. James K., HaRgreave T.B. : Immunosuppression by seminal plasma and possible clinical signifiance. Immunol. Today, 1984, $5: 357-363$.
29. Kaipia A., Toppari J., Huhtaniemi I., Paranko J. : Sex difference in the action of activin-A on cell proliferation of differentiating rat gonad. Endocrinology, 1994, $134: 2165-2170$.

30. KHAN S.A., NIESCHLAG E. : Interleukin-1 inhibits follitropin-induced aromatase activity in immature rat Sertoli cells in vitro. Mol. Cell Endocrinol., $1991,75: 1-7$.

31. Kiersenbaum A.L. : Mammalian spermatogenesis in vivo and in vitro: a partnership of spermatogenic and somatic cell lineages. Endocr. Rev., 1994, $15: 116-134$.

32. Lee K.-O., OH Y., Giudice L.C., Cohen P., Peehl D.M., ROSENFELD E.G. : Identification of insulinlike growth factor-binding protein-3 (IGFBP-3) fragments and IGFBP-5 proteolytic activity in human seminal plasma: a comparison of normal and vasectomized patients. J. Clin. Endocrinol. Metab., 1994, 79 : 1367-1372.

33. Lin T., Haskell J., Vinson N., TerRacio L. : Direct stimulatory effects of insulin-like growth factor I on Leydig cell steroidogenesis in primary culture. Biochem. Biophys. Res. Commun., 1986, 137 : 950-956.

34. LiN T., Blaisdeld J., Haskell J.F. : Transforming growth factor-b inhibits Leydig cell steroidogenesis. . Biochem. Biophys. Res. Commun., 1987, 146 : 387-394.

35. Lin T., Calkins H., Morris P.L., Vale W.W., BarDIN C.W. : Regulation of Leydig cell function in primary culture by inhibin and activin. Endocrinology, $1989,125: 2134-2140$.

36. Mallea L.E., Machado A.J., Navaroli F., RomMERTS F.F.G. : Epidermal growth factor stimulates lactate production and inhibits aromatization in cultured Sertoli cells from immature rats. J. Androl., 1986, $9: 201-208$.

37. Mather J.P., Attie K., Rice G., Phillips D. : Activin stimulates spermatogonial proliferation in Sertoli-germ cell co-cultures from immature rat testis. Endocrinology, 1990, 127 : 3206-3214.

38. Matsul Y., Toksoz D., Nishikawa S., et al. : The effect of steel factor, the ligand for c-kit and LIF/DIA on murine primordial germ cells in culture. Nature, 1991, $353:$ 750-752.

39. Mauduit C., Chauvin M.A., De Perrettil E., MoreRA A.M., Benahmed M. : Effect of activin A on dehydroepiandrosterone and testosterone secretion by primary immature porcine Leydig cells. Biol. Reprod., 1991, 45 : 101-109.

40. Mauduit C., Hartmann D.J., Chauvin M.A., Revol A., Morera A.M., BENAHMED M. : Tumor necrosis factor a inhibits gonadotropin action in cultured porcine Leydig cells: site(s) of action. Endocrinology, 1991, $129:$ 2933-2940. 
41. Mauduit C., Chauvin M.A., Hartmann D.J., Revol A., MoRera A.M., BENAHMED M. : Interleukin-1a as a potent inhibitor of gonadotropin action in porcine Leydig cells: site(s) of action. Biol. Reprod., 1992, 46 : 1119-1126.

42. Maudut C., Benahmed M. : Growth factors in the testis function and development. In : R. M. S Hamamah. Research in Male gametes: production and quality. Paris, Les Editions INSERM, 1996.

43. Mclachlan R.I., Robsertson D.M., DeKrester D.M., Burger H.G. : Advances in the physiology of inhibin and inhibin-related peptides. Clin. Endocrinol., 1988, $29: 77-114$.

44. Mealy K., Robinson B., Millette C.F., Majzoub J., WILMORE D.W. : The testicular effects of tumor necrosis factor. Ann. Surg., 1990, 211 : 470-475.

45. Mita M., Borland K., Price J.M., Hall P.F. : The influence of insulin and insulin-like growth factorI on hexose transport by Sertoli cells. Endocrinology, 1985, $116: 987-992$.

46. Morera A.M., Cochet C., Keramidas M., Chauvin M.A., De Peretti E., Benahmed M. : Direct regulating effects of transforming growth factor beta on the Leydig cell steroidogenesis in primary culture. J. Steroid Biochem., 1988, 30 : 443-447.

47. Murono E.P., WashbuRn. A.L. : Fibroblast growth factor inhibits 5a-reductase activity in cultured immature Leydig cells. Mol. Cell Endocrinol., 1990, 68 : R19- R23.

48. MurPhy P.R., Moger W.H. : Short-term primary culture of mouse interstitial cells: effects of culture conditions on androgen production. Biol. Reprod., 1982, $27: 38-47$.

49. NAZ R.K., KumaR R. : Transforming growth factor b1 enhances expression of a $50 \mathrm{kDa}$ protein related to 2'-5' oligoadenylate synthetase in human sperm cells. J. Cell Physiol., 1991, 146 : 156-163.

50. NAZ R.K., KAPLAN P. : Effects of epidermal growth factor on human sperm cell function. J. Androl, $1993,14: 240-7$.

51. NAZ R.K., KAPLAN P. : Increased levels of interleukin-6 in seminal plasma of infertile men. J. Androl., 1994, $15: 220-227$.

52. Naz R.K., Chaturvedi M.M., AgGarwal B.B. : Role of cytokines and proto-oncogenes in sperm cell function: relevance to immunologic infertility. Am. J. Reprod. Immunol., 1994, $32: 26-37$.

53. NAZ R.K., Minhas B.S. : Enhancement of sperm function for treatment of male infertility. $\mathrm{J}$. Androl., 1995, $16: 384-388$.
54. Nehar D., Mauduit C, Revol A., Morera A.M., BEHAHMED M. : Effect of epidermal growth factor/transforming growth factor alpha on lactate production in porcine Sertoli cells: glucose transport and lactate dehydrogenase isozymes as potential sites of action. Mol. Cell Endocrinol., 1993, 92 : 45-53.

55. Nehar D., Mauduit C., Boussouar F., Benahmed M. : Tumor Necrosis Factor a-stimulated lactate production is linked to an increase in lactate dehydrogenase A expression and activity in porcine cultured Sertoli cells. Endocrinology, 1997, sous presse.

56. Nocera M., CHU T.M. : Transforming growth factor-b as an immunosuppressive protein in human seminal plasma. Am. J. Reprod. Immunol., 1993, $30: 1-8$.

57. Noguchi S., OHBa Y., OKa T. : Involvement of epidermal growth factor deficiency pathogenesis of oligozoospermia in streptozotocin-induced diabetic mice. Endocrinology, 1990, $127: 2136-2140$.

58. Novero V., Camus M., Tournaye H., et al. : Relationship between serum follicle stimulating hormone in the male and standard sperm parameters, and the results of intracytoplasmic sperm injection. Hum. Reprod., 1997, $12: 59-63$.

59. OONK R.B., Grootegoed J.A. : Insulin-like growth factor-I (IGF-I) receptors on Sertoli cells from immature rats and age dependent testicular binding of IGF-I and insulin. Mol. Cell Endocrinol., $1988,55: 33-43$.

60. Ovesen P., Flyvbjerg A., Orskov H. : Insulin-like growth factor I (IGF-I) and IGF binding proteins in seminal plasma before and after vasectomy in normal men. Fertil. Steril., 1995, 63 : 913-918.

61. Parvinen M., Soder O., Pekka M., Froysa B., RitZEN E.M. : In vitro stimulation of stage-specific deoxyribonucleic acid synthesis in rat seminiferous tubule segments by interleukin-1a. Endocrinology, 1991, $129: 1614-1620$.

62. Parvinen M., Pelto-Huikko M., Soder O., et AL. : Expression of b-nerve growth factor and its receptor in rat seminiferous epithelium: specific function at the onset of meiosis. J. Cell Biol., 1992, 117 : 629-641.

63. Pesonen K., Vinikka L., Koskimies A., Banks A.R., Nicolson M., Peheentupe J. : Size heterogeneity of epidermal growth factor in human body fluids. Life Sci., 1987, 40 : 2489-2494.

64. Pollanen P., Soder O., Parvinen M. : Interleukin1 alpha stimulation of spermatogonial proliferation in vivo. Reprod. Fertil. Dev., 1989, 1 : 85-87.

65. Rajasekaran M., Hellstrom W.J.G., Naz R.K., SIKKA S.C. : Oxidative stress and interleukins in seminal plasma during leukocytospermia. Fertil. Steril., 1995, 64 : 166-171. 
66. Ramasharma K., Cabrera C.M., Li C.H. : Identification of insulin-like growth factor-II in human seminal and follicular fluids. Biochem. Biophys. Res. Commun., 1986, 140 : 536-540.

67. Robertson D.M., Risbridger G.P., Hedger M., MCLACHLAN R.I. : Growth factors in the control of testicular function. In : D. deKretser. Molecular biology of the male reproductive system. San Diego, Academic Press, 1993 : 411-438

68. Russell L.D., Weiss T., GoH J.C., CurL J.L. : The effects of submandibular gland removal on testicular and epididymal parameters. Tiss. Cell, 1990, $22: 263-268$.

69. Sanderson N., Factor V., Nagy P., eT AL. : Hepatic expression of mature transforming growth factor b1 in transgenic mice results in multiple tissue lesions. Proc. Natl. Acad. Sci. U. S .A., 1995, 92 : 2572-2576.

70. SAndlow J.I., Feng H.-L., Cohen M.B., SANdRA A. : Expression of c-kit and its ligand, Stem Cell Factor, in normal and subfertile human testicular tissu. J. Androl., 1996, $17: 403-408$.

71. SHARPE R.M. : Regulation of spermatogenesis. In : N. J. Knobil E. The Physiology of Reproduction. New York, Raven Press, 1994 : pp 1363-1434

72. Shimoya K., Matsuzkr N., IDA N., ET AL. : Detection of monocyte chemotactic and activating factor (MCAF) and interleukin (IL)-6 in human seminal plasma and effect of leukospermia on these cytokine levels. Am. J. Reprod. Immunol., 1995, 34 : 311316.

73. Soder O., Bang P., Wahab A., Parvinen M. : Insulin-like growth factors selectively stimulate spermatogonia, but not meiotic deoxyribonucleic acid synthesis during rat spermatogenesis. Endocrinology, 1992, 131 : 2344-2350.

74. Sordoillet C., Chauvin M.A., Revol A., Morera A.M., BENAHMED M. : Fibroblast growth factor is a regulator of testosterone secretion in cultured immature Leydig cells. Mol. Cell. Endocrinol., $1988,58: 283-286$.

75. Sordoillet C., Chauvin M.A., de Peretti E., MoRerA A.M., BENAHMED M. : Epidermal growth factor directly stimulates steroidogenesis in primary culture of porcine Leydig cells: actions and sites of action. Endocrinology, 1991, $128: 2160$ 2168.

76. Sordolllet C., Chauvin M.A., Hendrick J.C., Franchimont P., Morera A.M., Benahmed M. : Sites of interaction between epidermal growth factor and transforming growth factor-beta 1 in the control of steroidogenesis in cultured porcine Leydig cells. Endocrinology, 1992, 130 : 13521358.
77. Sundel A., LiabakK N., Austgulen R., Espevik T. : High concentrations of the soluble tumor necrosis factor receptor p55 in human seminal plasma. International Symposium on Male Infertility and Assisted Reproduction. April 21-24 1993, Genk, Belgium.

78. Syed V., KhaN S.A., NieschlaG E. : Epidermal growth factor stimulates testosterone production of human Leydig cells in vitro. J. Endocrinol. Invest., 1991, $14: 93-97$.

79. Tournaye H., Verheyen G., Nagy P., et al. : Are there any predictive factors for successful testicular sperm recovery in azoospermic patients? Hum. Reprod., 1997, $12: 80-86$.

80. Tsutsumi O., Kurachi H., OKa T. : A physiological role for epidermal growth factor in male reproductive function. Science, 1986, 233 : 975-977.

81. Van Dissel-Emiliani F.M.F., Grootenhuis A.J., De JoNG F.H., DE RooIJ D.G. : Inhibin reduces spermatogonial numbers in testes of adult mice and chinese hamster. Endocrinology, 1989, 125 : 18981903.

82. Verhokven G., Caildeau J. : Stimulatory effects of epidermal growth factor on steroidogenesis in Leydig cells. Mol. Cell Endocrinol., 1986, 47 : 99-106.

83. WARTENBERG $\mathbf{H}$. : Differentiation and development of the testes. In : d. K. D. Burger H. The testis. New York, Raven Press, 1989 : 67-118.

84. Xiong Y., Hales D.B. : The role of tumor necrosis factor-alpha in the regulation of mouse Leydig cell steroidogenesis. Endocrinology, 1993, $132: 2438$ 2444.

85. Yamamoto M., Miyake K. : Successful use of interferon for male infertility. Lancet, 1994, $34: 614$.

86. Yie S.-M., LobB D.K., ClaRK D.A., Younglai E.V. : Identification of a transforming growth factor alpha-like molecule in human seminal plasma. Fertil. Steril., 1994, 61 : 129-135.

\section{ABSTRACT}

GROWTH FACTORS AND CYTOKINES IN THE PHYSIOLOGY AND PATHOLOGY OF MALE GONADE

\author{
C. Mauduit, S. Hamamah, B. Loras, \\ M. BENAHMED
}

Since 4 years ago, intracytoplasmique sperm injection (ICSI) represents a new therapeutic approach of different forms of male sterility including obs- 
tructives and spermatogenic failure azoospermia. However, the evaluation of the male infertility begins with the study of semen characteristics particularly sperm count and motility assessment. However, testicular activites are not only under the control of the neuroendocrine system but also under that of local growth factors and cytokines.

In this review, we report, in the first part, the different observations indicating the key role of growth factors and cytokines in the fetal testis development as well as in the differentiated functions, steroidogenesis and gametogenesis. In a second part, we report the data, obtained by different teams, related to the evaluation and/or actions of these factors in infertile men. Moreover, the low number of data related to the use of these factors in order to improve sperm fuction are mentioned. Finally, in a prospective context, we indicate some points which seem of interest for elucidating some aspects of growth factor and cytokine role in fertility/infertility.

Key words : Testis, growth factors, cytokines, male infertility, oligo and azoospermia. 